\title{
Nanostructure of thin silicon films by combining HRTEM, XRD and Raman spectroscopy measurements and the implication to the optical properties
}

\author{
Andreja Gajović ${ }^{\text {a,*, Davor Gracin a, Igor Djerdj }}{ }^{\text {b,c }}$, Nenad Tomašić ${ }^{\text {d }}$, Krunoslav Juraić a \\ Dang Sheng Su ${ }^{\mathrm{e}}$
}

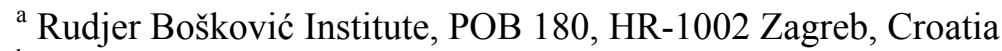

${ }^{\mathrm{b}}$ ETH Zürich, Department of Materials, Zürich, Switzerland

${ }^{c}$ Department of Physics, Faculty of Science, University of Zagreb, POB 331, HR-10002 Zagreb, Croatia

${ }^{\mathrm{d}}$ Institute of Mineralogy and Petrography, Faculty of Science, University of Zagreb, Horvatovac bb, HR10000 Zagreb, Croatia

${ }^{\mathrm{e}}$ Fritz Haber Institut der Max-Planck-Geselschaft, Faradayweg 4-6, D-14159 Berlin, Germany

* Corresponding author. Tel.: +385 14561106; fax: +385 14680112, e-mail address: gajovic@irb.hr

\begin{abstract}
A series of thin silicon films with different degrees of crystallinity were prepared by decomposition of silane gas highly diluted with hydrogen, in radiofrequency glow discharge. The crystallite size, shape, and the portion of crystalline phase were investigated by highresolution transmission electron microscopy (HRTEM), selected area electron diffraction (SAED), Raman spectroscopy (RS), and X-ray powder diffraction (XRD). The absorption coefficient (a) was calculated from the measurement of UV-vis-transmittance. By using RS, the volume fractions of the crystalline phase were estimated from the ratio of the integrated intensities of transversal optical (TO)-related crystalline and amorphous bands. These results were in excellent agreement with the mean crystallite sizes measured in HRTEM images and crystallite sizes refined from XRD measurements. The red shift of absorption, appearing as a result of the increase of the crystal fraction, depends on the size and distribution of nanocrystals.
\end{abstract}

Keywords: Silicon; Solar cells; Nanostructures; Optical properties

\section{Introduction}

Crystalline silicon (c-Si) is an appropriate semiconductor material for simple single element terrestrial photovoltaic cells, since silicon is non-toxic, abundant in the Earth's crust (25\%) having an almost ideal band-gap $(1.1 \mathrm{eV})$ and long-term stability [1]. To avoid the high costs to produce high-quality c- Si solar cells, amorphous (a-Si) thin films, a direct band-gap material, has been used to fabricate solar cells for several decades. Due to the high absorption coefficient in the visible part of solar radiation, even $300 \mathrm{~nm}$ of the material is sufficient for a reasonably efficient solar cell. However, a-Si is not a stabile structure [2] since the prolonged illumination introduces defects that decrease the initial solar cell efficiency [3]. The nanocrystalline material (nc-Si) is much more stable and has broader absorption range in the visible part of the solar radiation [4,5]. Furthermore, due to quantum effects it is possible to change the optical gap by changing the size of nanocrystals [6]. These properties make thin silicon films, with a mixture of amorphous and nanocrystalline structures, a promising candidate for the third generation of solar cells, i.e., low cost solar cells with an efficiency above $30 \%[7,8]$.

One of the common techniques in large-scale commercial production nc-Si thin film for solar cells is plasma-enhanced chemical vapor deposition (PECVD) by decomposition of silicon hydrides in a radiofrequency glow discharge $[1,9]$. To optimize the production parameters in order to improve the properties of prepared nc-Si thin film layers, the structure of the films has to be systematically studied and furthermore correlated to the characteristics relevant for their application. Thus, we study the correlation between nanostructural and optical properties of nc $\mathrm{Si}$ produced by PECVD. In order to find reliable procedure to determinate the nanostructural 
properties, we compared results obtained by microscopic techniques with Raman and X-ray diffraction (XRD) as representatives of "bulk probe" techniques.

High-resolution transmission electron microscopy (HRTEM) and selected area electron diffraction (SAED) at nanostructured Si thin films was shown as amethod of choice in crystal structure determination of Si ordered domains of $1-5 \mathrm{~nm}$ in size [10,11]. Moreover, the size distribution, interplanar distance, lattice parameter, and lattice expansion of the individual Si nanocrystals in porous silicon (PS) formed by the electrochemical etch of boron-doped ( $p$-type) silicon wafers was also extensively studied by HRTEM [12,13,14].

Nevertheless, the sample area observed by (HR)TEM presents quite local properties and the statistically more reliable microstructure analysis is necessary for the evaluation of structure on "large area", especially when the samples of industrial production were considered. As the "bulk probe method" of structural study, XRD is usually provided, mostly using the Sherrer formula to estimate the Si nanocrystal size from the line broadening, assuming only one reflection, (1 11 1), [6,9,15-18]. An advanced analysis of XRD spectra was done byMartín Palma et al. [19] by usingMonteCarlo interference-function-fitting algorithm to determine the distribution in size of the Si grains that compose PS as a function of porosity. For the purpose of this work, we used XRD and Rietveld refinement for estimation the crystal to amorphous fraction and crystal size.

Raman spectroscopy (RS) is non-destructive, extensively employed technique for structural analysis of completely amorphous or amorphous-nanocristalline multicomponent silicon-based thin films [4,15,20-24]. Since the interpretation of the Raman measurements largely influences the estimated structural data, RS has to be combined with other methods, typically microscopy or XRD. Manotas et al. [25] combined microphotoluminescence and micro-Raman spectra taken on the cross-section of porous silicon multilayers to get direct depth-resolved information, but the spatial resolution of these methods cannot reach nanometric scale. RS spectroscopy results were combined with grazing incidence small angle Xray scattering (GISAXS) results as well, but the crystals seen by RS and the "particles" seen by GISAXS are not necessarily the same objects [22].

The most reliable results can be achieved only by combination of "local" and "bulk" probe methods. Thus, Moutinho et al. [9] combined TEM, RS and XRD results but only in order to identify phases of the thin film without any quantitative analysis and/or correlation of the results obtained by different techniques.

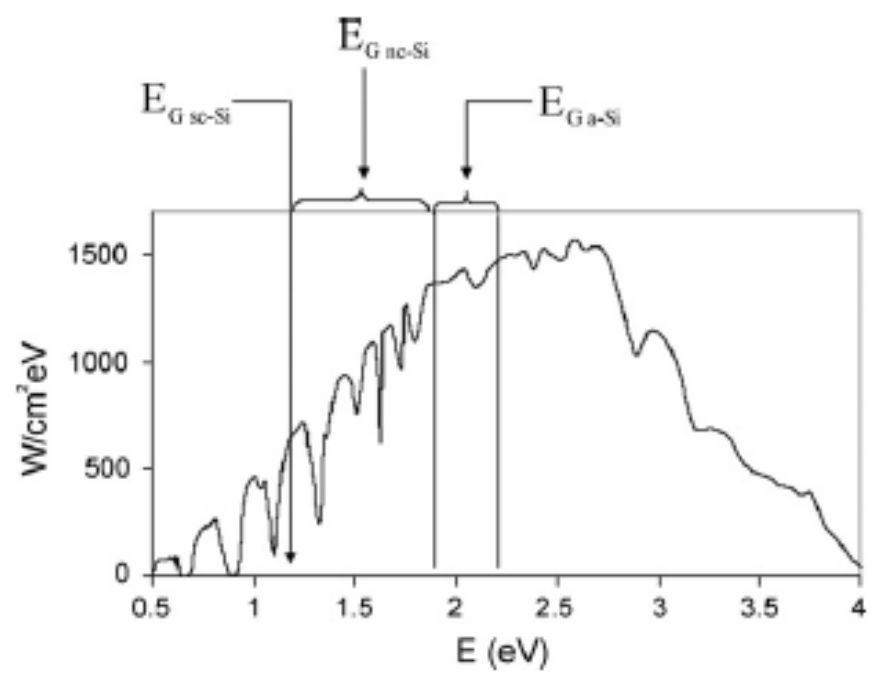

Fig. 1. Solar irradiance spectra and energy gaps $\left(E_{G}\right)$ of different silicon structural forms: single-crystalline silicon (sc-Si), nanocrystalline silicon (nc-Si), and amorphous silicon (a-Si). 
In this work we perform the detailed study of thin Si films nanostructure and its influence on the optical properties of these Si thin films following the scheme presented in Fig. 1. Single crystal Si has energy gap exactly at $1.1 \mathrm{eV}\left(\mathrm{E}_{\mathrm{G} \mathrm{sc-Si}}\right.$ in Fig. 1), whereas amorphous Si thin films have energy gaps in the region between 1.9 and $2.2 \mathrm{eV}\left(\mathrm{E}_{\mathrm{G} \text { a-Si }}\right.$ in Fig. 1). Furthermore the nanocrystals added in the structure of the amorphous thin Si films enlarged the region of the energy gaps in much broader part of the solar irradiant spectra covering the energy region between 1.1 and 1.9 $\mathrm{eV}\left(\mathrm{E}_{\mathrm{G}} \mathrm{nc}-\mathrm{Si}\right.$ in Fig. 1) thus enabling an increase of thin film solar cells efficiency. The nanostructure of prepared thin films was systematically studied by bringing together and evaluating the results obtained from fundamentally different experimental techniques; RS, Rietveld refinement of XRD and HRTEM. Moreover, the possibility of tailoring the optical absorption regarding the structure and in that way increasing the solar cell efficiency in multicomponent structures was demonstrated.

\section{Experimental}

Thin Si films, with thicknesses of about $100 \mathrm{~nm}$, were deposited on a $3 \mathrm{~mm}$ thick glass substrate by radio frequency plasma-enhanced chemical vapor deposition (RF PECVD) in a capacitively coupled planar diode source. Radio frequency applied in these experiments was $13.6 \mathrm{MHz}$. The working gas was silane diluted with hydrogen in the range $5-7 \%$ of silane in the gas mixture while the radio frequency (RF) plasma power densities were varied between 10 and $15 \mathrm{~mW} \mathrm{~cm}^{-2}$.

The growing conditions were selected to obtain different degrees of crystal fractions and a variety in individual crystal sizes. More details about growing conditions can be found in our previous papers [22,24]. As a rule, the higher the silane dilution, the larger the crystals and higher fraction of crystalline phase are obtained.

Raman spectroscopy was performed using computerized DILOR Z24 triple monochromator with Coherent INNOVA 400 argon ion laser, operating at $514.5 \mathrm{~nm}$ line for excitation. Anaspec's doublepass prism premonochromator was used to reduce parasite laser plasma lines. The incident laser beam was focused to the line shape of $50 \mu \mathrm{m}$ x $3000 \mu \mathrm{m}$, thus the laser power density of $100 \mathrm{~W} \mathrm{~cm}^{-2}$ was applied to avoid heating and/or crystallization of the samples in the laser beam during the recording of the spectra. Recording of the spectra was performed in vacuum to avoid the observation of rotation lines of the air superimposed to the amorphous silicon bands of low intensity. The typical resolution was between 1 and $2 \mathrm{~cm}^{-1}$. When the Raman spectra were analyzed, the ratio of areas under corresponding transversal optical (TO) phonon peaks in Raman was taken as a measure of crystalline to amorphous fraction while variation of TO peak positions, $\omega_{\mathrm{TO}}$, was taken as an estimation of crystal size using the empirical formula [21]:

$$
d_{\mathrm{RAMAN}}=2 \pi\left(\frac{2}{\left(522-\varpi_{\mathrm{TO}}\right)}\right)^{1 / 2}
$$

The XRD data were obtained by a Philips PW 3040/60 X'Pert PRO powder diffractometer using $\mathrm{Cu}$ Ka radiation $(\lambda=1.54055 \AA)$ at $45 \mathrm{kV}$ and $40 \mathrm{~mA}$. The incident beam was passed through an X-ray mirror having a divergence slit of $0.30^{\circ}$. The diffracted beam was directed to the detector through a parallel plate collimator with equatorial acceptance angle of $0.18^{\circ}$. The samples were mounted on a single silicon crystal disk cut in a manner to avoid lattice planes, and thus provide no silicon diffraction and low background. The disk with a sample was inserted into the sample spinner programmed to a revolution time of $1 \mathrm{~s}$. Step size was set to $0.02^{\circ}$ with a measuring time of $2 \mathrm{~s}$ per step.

A modified Rietveld refinementwas used for the calculation of crystalline fractions by adding 10 wt.\% of $\mathrm{SiO}_{2}$ to the samples. The added $\mathrm{SiO}_{2}$ served as an internal standard because its diffraction lines were narrow and it had the same linear absorption coefficient for incident X- 
rays as the examined samples. Therefore, there was no need to make the absorption correction. When an internal standard phase, $\mathrm{s}$, is added to the mixture in the weight fraction Ws, the absolute weight fractions of the other identified components, $p$, are given by Eq. (2):

$$
W_{p}=\frac{W_{\mathrm{s}} W_{p}^{r}}{W_{\mathrm{s}}^{r}}
$$

where superscript $r$ denotes the refined value of weight fraction within the Rietveld procedure and Hill and Howard method [26]. In this case, a shortfall from unity of the sum of the determined weight fractions of the $n$ identified components provides an estimate of the amount of crystalline to amorphous component in the sample.

HRTEM and SAED were performed on a Philips CM200 FEG microscope with a field emission gun operating at $200 \mathrm{kV}$. The samples were polished and thinned by an ion beam for transmission electron microscopy. Distribution of the crystallite sizes was calculated using the software Statistica [27]. Filtering of the HRTEM images was performed using the program CRISP to illustrate the crystallinity of Si films, apparently hidden in the amorphous matrix, and to detect the presence of defects in the structures.

Optical constants (index of refraction and absorption coefficient) and film thickness were calculated from transmittance data in the UV-vis-NIR part of spectra, using pointwise unconstrained optimization approach (PUMA) [28]. We considered a thin film, deposited on a thick transparent substrate (glass).

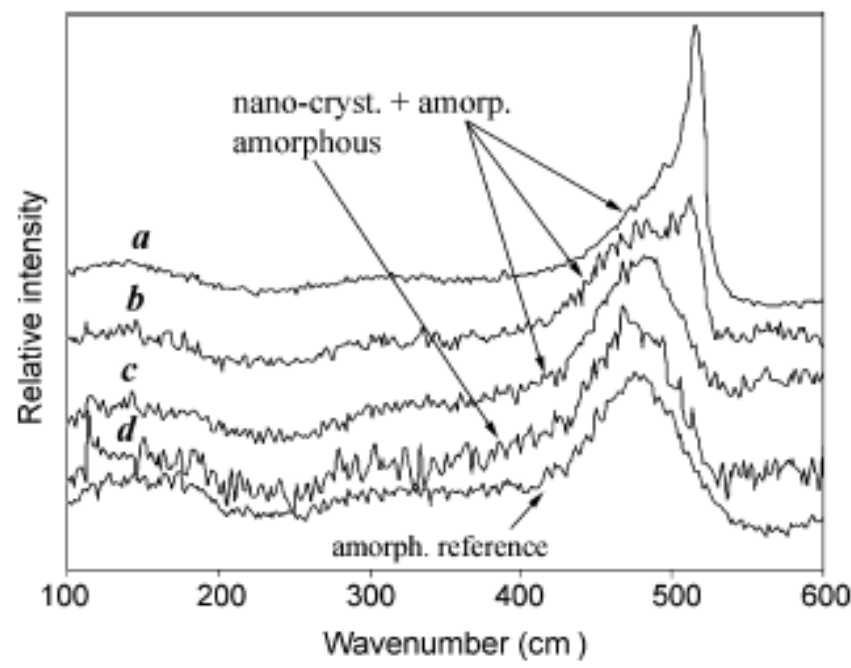

Fig. 2. Raman spectra of thin film silicon samples with different degrees of crystallinity.

\section{Results and discussion}

Fig. 2 shows the Raman spectra of a series of deposited samples, with different degree of crystallinity, from about $30 \%$ of crystalline phase to pure amorphous phase.

Silicon has a cubic structure, space group Fd3m with one Raman active mode of vibration. The microcrystalline $\mathrm{Si}$ is characterized by one intensive sharp band in Raman spectra. The position of this band is $520 \mathrm{~cm}^{-1}$, it is broadened and shifted to lower frequencies for crystallites smaller than $30 \mathrm{~nm}$, and nanocrystallinity is also manifested by low-energy shoulder [6]. The broad band with maxima around $480 \mathrm{~cm}^{-1}$ is attributed to amorphous phase [29]. The volume fraction of the crystalline phase used to be estimated from the ratio of the integrated intensities of crystalline and amorphous bands after deconvolution of the spectra.

Values for the crystalline fraction of the investigated samples, obtained from the ratio of corresponding TO bands, and the average individual crystal size are listed in Table 1. However, 
the process of deconvolution of the Raman bands is not straightforward due to the partial overlapping of the bands. Moreover, quantitative determination of crystallinity only from Raman measurements is approximate because of different cross-section factors of Raman scattering for crystalline and amorphous silicon; so the results were compared with XRD measurements as well as with HRTEM observations.

Table 1

Crystalline fraction and average crystallite sizes estimated from RS

\begin{tabular}{|l|l|l|}
\hline Sample & Crystalline fraction (\%) & Crystallite size $(\mathrm{nm})$ \\
\hline $\mathrm{a}$ & $29 \pm 2$ & $6-8$ \\
\hline $\mathrm{b}$ & 15 & 2.9 \\
\hline $\mathrm{c}$ & 8 & 1.8 \\
\hline $\mathrm{d}$ & Amorphous & \\
\hline
\end{tabular}

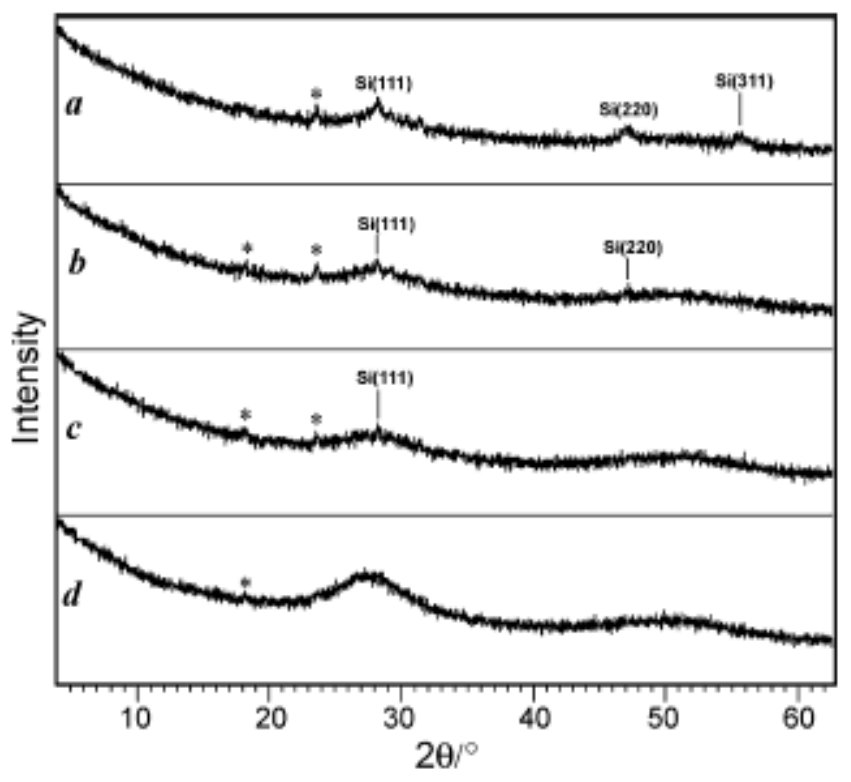

Fig. 3. XRD of thin silicon film samples * parasite lines.

XRD measurements were used as the other technique for a more quantitative determination of crystallinity. XRD patterns of the studied samples are shown in Fig. 3. The broad amorphous halo was observed for all samples. A sample with significant amount of crystalline phase exhibits narrow diffraction lines superimposed to the broad amorphous halo. In sample a (Fig. 3), the diffraction lines of $\left(\begin{array}{lll}1 & 1 & 1\end{array}\right),\left(\begin{array}{lll}2 & 2 & 0\end{array}\right)$, and $\left(\begin{array}{lll}3 & 1 & 1\end{array}\right)$ are clearly observed indicating a considerably higher amount of crystalline phase than in the other samples. In samples b and c (Fig. 3), (lll 111$)$ and (2 20 ) lines are hardly observable, while sample d (Fig. 3) is completely X-ray amorphous.

The weight fraction of amorphous phase and average crystallite sizes were calculated using the modified Rietveld refinement of XRD patterns with $\mathrm{SiO}_{2}$ as internal standard. For the sample with a higher amount of crystalline phase (Fig. 3a), the calculated weight fraction of crystalline phase was $25.2 \%$ and average crystallite size $11.1 \mathrm{~nm}$, thus in agreement with the results estimated from Raman spectrum. For other samples, the refinement showed uncertain results due to a very small amount of crystalline phase.

XRD is less efficient than electron diffraction, especially at nanoscale. SAED had shown diffraction spots in all investigated samples (Fig. 4). The appearance of electron diffraction spots 
in sample d (Fig. 4d) indicates a small amount of crystallites in that sample and/or the crystallite sizes under the detection limit of the XRD or RS technique. Observed diffraction spots were superimposed to amorphous halo, further demonstrating the presence of both amorphous and crystalline phases in the studied thin silicon films.

High-resolution transmission electron microscopy (HRTEM) was used for direct analysis of the sizes and the shapes of crystallites. Although in one sample (sample d), RS and XRD results did not reveal any crystalline bands/lines, HRTEMimages showed crystallites with different sizes and shapes in all samples. Fig. 5 shows selected HRTEM images for the studied samples. Crystallite sizes were measured in several HRTEM images for every sample to achieve better statistics. Distributions of particle sizes (Fig. 6) were fitted to log-normal distribution and statistical parameters are listed in Table 2. The samples a and b (Fig. 6 and Table 2) show broader distribution of particle sizes than the other samples. The mean crystallite sizes observed in XRD and RS amorphous sample $\mathrm{d}$ is $1.89(6) \mathrm{nm}$, which is larger than that in sample c. However, a very small amount of crystallites was observed in sample $d$ and most of them were under detection limit of XRD and RS measurements.
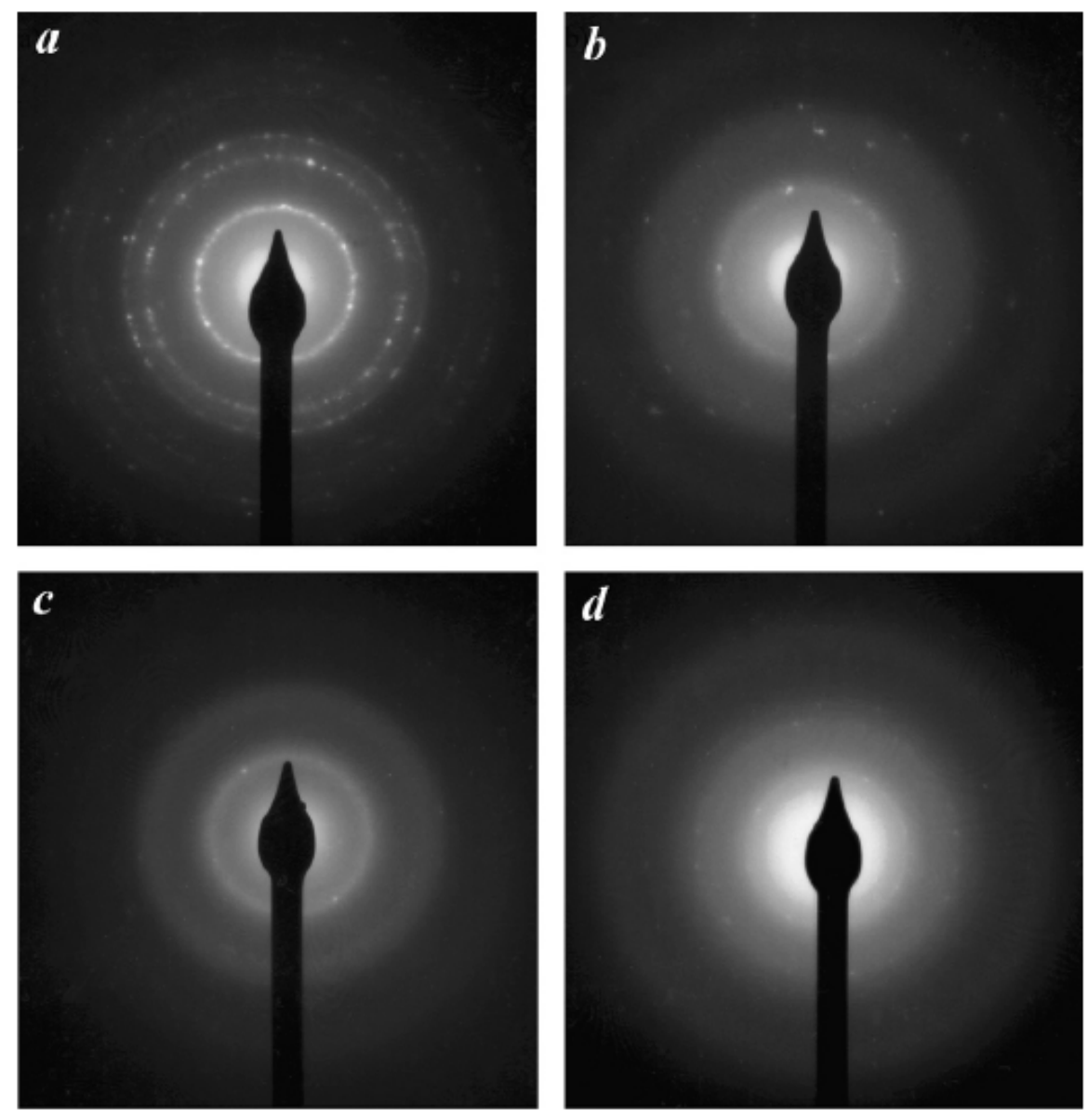

Fig. 4. SAED of thin silicon film samples.

Filtering of HRTEM images was done in order to achieve a better recognition of the crystallites in the samples, to get insight into their arrangement, and to detect the eventual presence of crystallite defects. Information about crystal structure defects is important, because defects and strain can influence Raman results. For illustration, two filtered regions of the HRTEMimage of sample a are shown in Fig. 7. In the power spectrum (Fourier transform) of the image, numerous spots of crystalline Si are present thus indicating a high amount of crystallites. In the filtered image of the region 1 (Fig. 7), some dislocation arrays in the crystallites are evident, while the 
filtered image of the region 2 shows the arrangement of crystallites with different orientations with respect to each other.
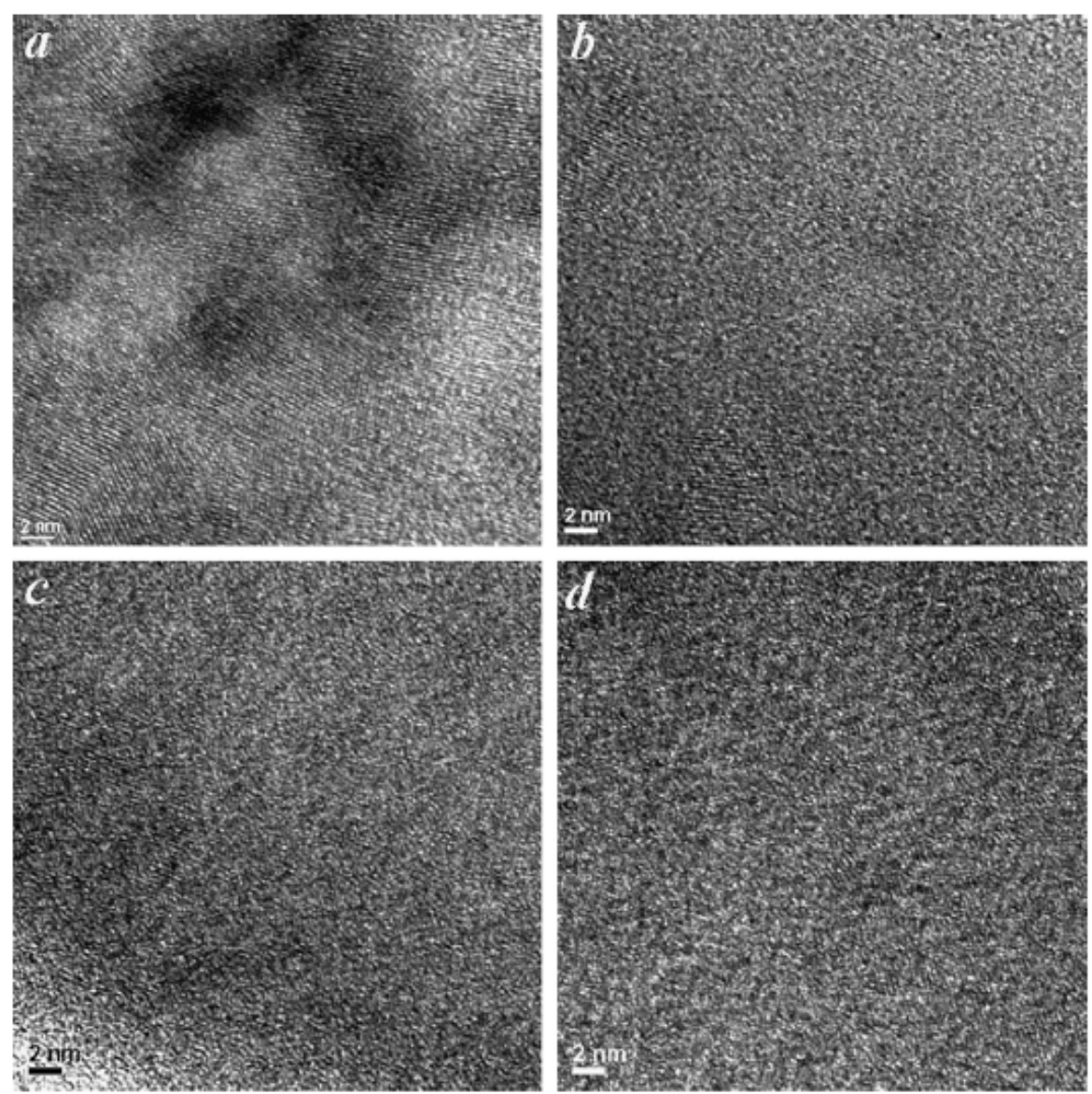

Fig. 5. HRTEM of thin silicon film samples.
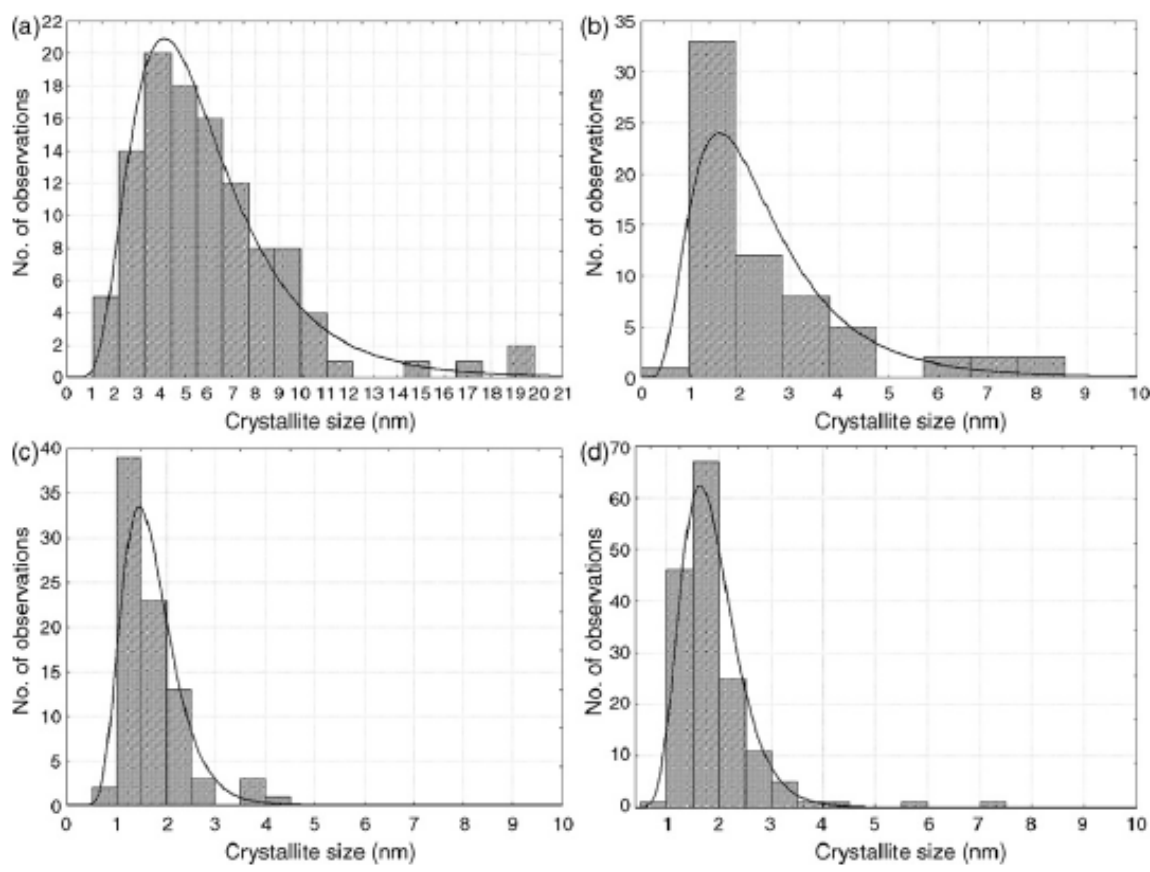

Fig. 6. Distribution of the crystallite sizes measured in HRTEM images and fitted to log-normal distribution. 
Table 2

Crystallite sizes measured in HRTEM images and statistical parameters for lognormal distribution fitting

\begin{tabular}{|l|l|l|l|l|l|l|}
\hline Sample & Mean(nm) & Median(nm) & Min.(nm) & Max.(nm) & Variance(nm $\left.{ }^{2}\right)$ & S.D.(nm) \\
\hline a & 6.0 & 5.4 & 1.62 & 19.8 & 10.6 & 3.2 \\
\hline b & 2.5 & 1.7 & 0.9 & 8.4 & 2.9 & 1.7 \\
\hline c & 1.72 & 1.54 & 0.99 & 4.38 & 0.43 & 0.65 \\
\hline d & 1.89 & 1.75 & 0.93 & 7.13 & 0.56 & 0.75 \\
\hline
\end{tabular}
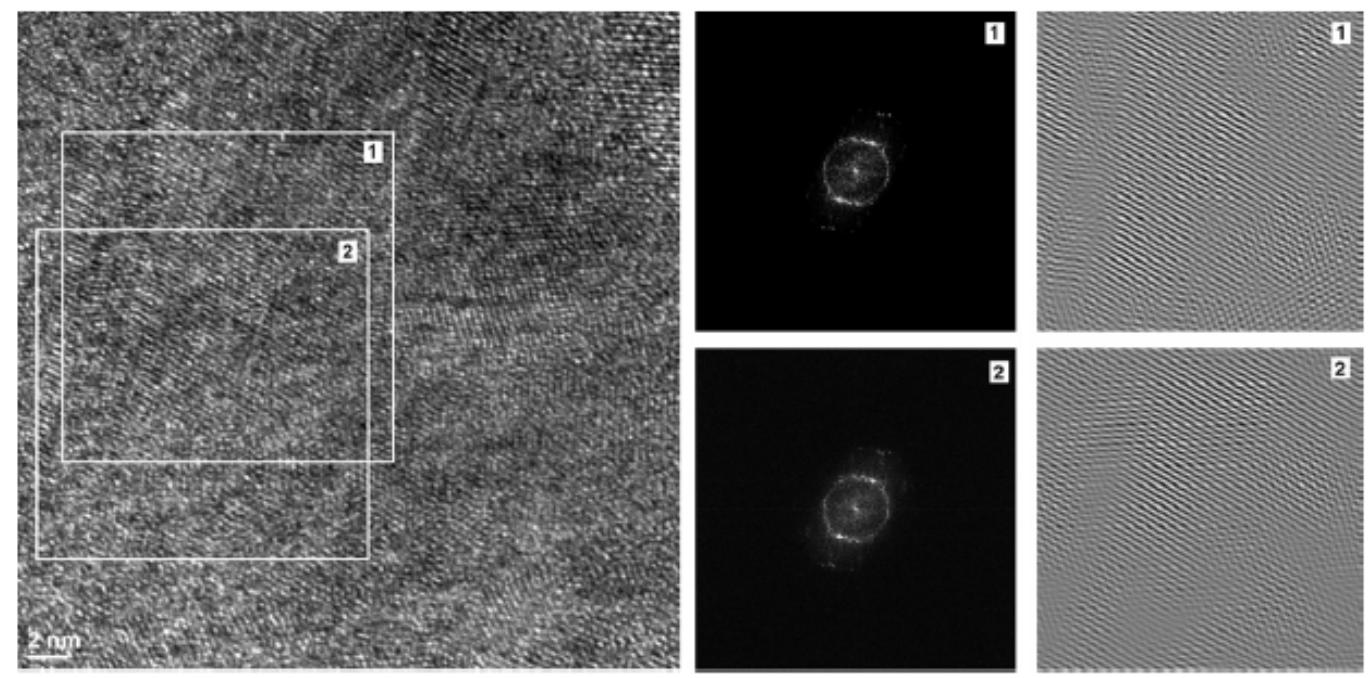

Fig. 7. Filtering of the HRTEM image of sample a.

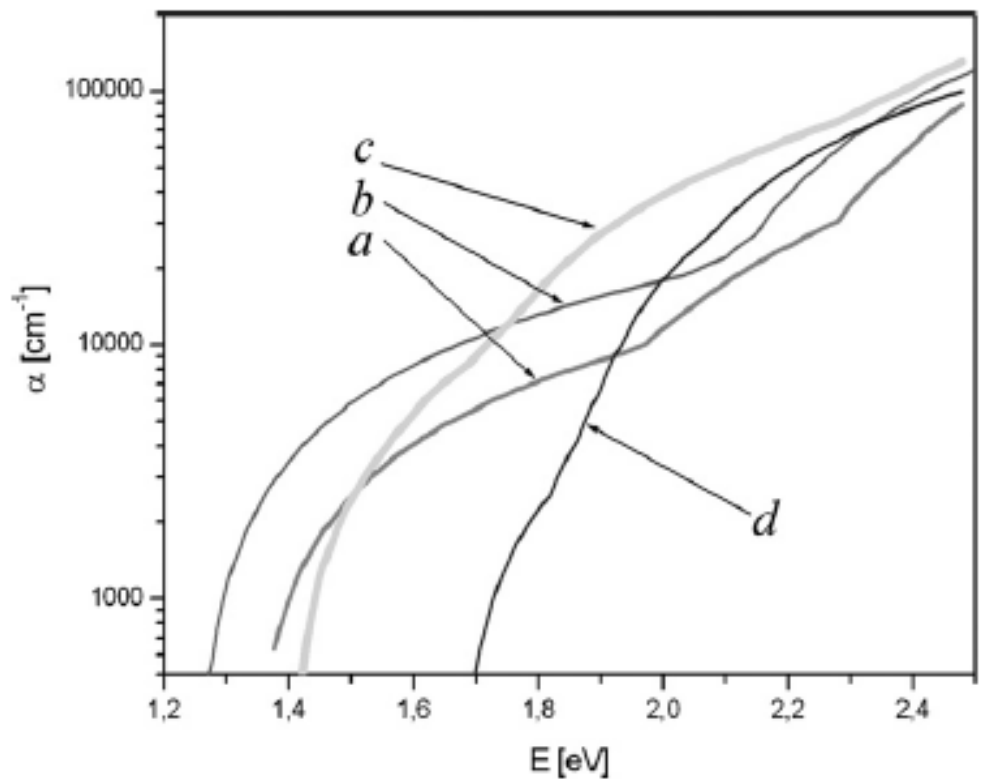

Fig. 8. Distributions of absorption coefficients (a) for thin silicon film samples.

Fig. 8 shows a comparison of energy distribution of the absorption coefficient $(\alpha)$ for amorphous and nanocrystalline samples with similar crystal fractions, of similar density but different size of crystals. The electrons in monocrystalline silicon follow an indirect optical band-to-band transition while for amorphous silicon this selection rule is relaxed. Consequently, XRD amorphous sample d has a dominant absorption at higher photon energy, between 2.0 and 2.4 
$\mathrm{eV}$. However, below some $1.8 \mathrm{eV}$, the sample is transparent. The absorption of sample with the largest grains, sample a, is similar to monocrystalline $\mathrm{Si}$; thus, its absorption is lower for higher energies while complete distribution of $\alpha$ has a red shift toward monocrystalline values. When comparing the energy distribution of solar spectrum (Fig. 1) with the distribution of $\alpha$ in Fig. 8, the optimal photovoltaic response in a single layer solar cell is expected from sample c, which is experimentally verified and the structure is characterized as "close to the amorphous to crystalline phase transition'. For multilayer solar cells, the structures containing different sizes and size distribution of nanocrystals should be combined to achieve best performance. For increasing solar cell efficiency at low energies, the samples a and b show obvious advantage. Their high $a$ in the broader energy region can be explained by comparing the results of energy distribution of $\alpha$ (Fig. 8) with crystallite sizes and their distributions (Fig. 6 and Table 2). Sample $b$ with average crystallite sizes smaller than $3 \mathrm{~nm}$ and with a large standard deviation of crystallite sizes has the higher $\alpha$ in the broader energy region. Moreover, this result indirectly proves that the energy gap of the $\mathrm{Si}$ is correlated to crystallite dimension (Fig. 1). The broader distribution of the small crystallite sizes causes broader energy distribution of the absorption coefficient.

\section{Conclusions}

The crystalline phase in the studied nanocrystalline thin silicon films estimated from RS was between 0 and 30\%. However, SAED showed reflections even in RS and/or XRD amorphous sample; thus, the high local amount of crystallites observed in HRTEM images has confirmed the presence of crystalline phase in all investigated materials. This result is due to the small amount of crystallites and small crystallite sizes that are under the direction limit of RS and XRD techniques.

The crystallite fraction and the average crystallite size calculated from XRD were in accordance with the results estimated from Raman spectra. Moreover, mean crystallite sizes measured in HRTEM images are in very good agreement with results estimated from RS for crystalline samples. The mutual agreement of the results obtained by completely different physical phenomena shows that the vibrational properties (TO phonon mode) of the investigated material are directly influenced by nanometric crystallite sizes due to quantum confinement.

Samples with broader distribution of crystallite sizes but with average crystallite sizes smaller than $3 \mathrm{~nm}$ showed higher absorption coefficients in the broader energy region. Thus, this work demonstrates the possibility of tailoring the optical absorption and in that way increasing the solar cell efficiency in various multilayer structures.

\section{Acknowledgments}

The work in Zagreb was supported by Croatian Ministry of Science Education and Sport, grants "Physics and applications of nanostructures and bulk matter" (098-0982904-2898), "The thin filmsilicon on theamorphous to crystalline transition" (098- 0982886-2894), "Structure and properties of novel nanomaterials prepared by modern techniques" (119-0982886-1009) and "Interaction of minerals and environment" (119-0000000-1158) and by the European Commission under the contract no. INCOCT- 2004-509178. The work in Berlin is financially supported by Max-Planck Society. 


\section{References}

[1] G. Aberle Armin, Thin Solid Films 511-512 (2006) 26.

[2] Z. Iqbal, S. Veprek, J. Phys. C 15 (1982) 377.

[3] D.L. Staebler, C.R. Wronski, Appl. Phys. Lett. 31 (1977) 292.

[4] Y. Xu, X. Liao, G. Kong, X. Zeng, Z. Hu, H. Diao, S. Zhang, J. Cryst. Growth 256 (2003) 27.

[5] J.P. Kleider, C. Longeaud, R. Bruggemann, F. Houzé, Thin Solid Films 383 (2001) 57.

[6] I.H. Campbell, P.M. Fauchet, Solid State Commun. 58 (1986) 739.

[7] M.A. Green, Mater. Sci. Eng. B 74 (2000) 118.

[8] M.A. Green, Third Generation Photovoltaics: Ultra-High Efficiency at Low Cost, Springer-Verlag, 2003.

[9] H.R. Moutinho, B. To, C.-S. Jiang, Y. Xu, B.P. Nelson, C.W. Teplin, K.M. Jones, J. Perkins, M.M. Al-Jassim, J. Vac. Sci. Technol. A 24 (1) (2006) 95.

[10] G. Viera, S. Huet, M. Mikikian, L. Boufendi, Thin Solid Films 403-404 (2002) 467.

[11] G. Conibeer, M. Green, R. Corkish, Y. Cho, E.-C. Cho, C.-W. Jiang, T. Fangsuwannarak, E. Pink, Y. Huang, T. Puzze, T. Trupke, B. Richards, A. Shalav, K.-L. Lin, Thin Solid Films 511-512 (2006) 654.

[12] R.J. Martín-Palma, L. Pascual, P. Herrero, J.M. Martínez-Duart, Appl. Phys. Lett. 81 (2002) 25-27.

[13] L. Pascual, R.J. Martín-Palma, A.R. Landa-Cánovas, P. Herrero, J.M. Martínez-Duart, Appl. Phys. Lett. 87 (2005) (251921-1-3).

[14] R.J. Martín-Palma, L. Pascual, A. Landa, P. Herrero, J.M. Martínez-Duart, Appl. Phys. Lett. 85 (2004) 2517.

[15] C.-Y. Lin, Y.-K. Fang, S.-F. Chen, S.-H. Chang, T.-H. Chou, Mater. Sci. Eng. B 134 (2006) 99.

[16] M. Morales, Y. Leconte, R. Rizk, D. Chateigner, Thin Solid Films 450 (2004) 216.

[17] K. Fukaya, A. Tabata, T. Mizutani, Thin Solid Films 478 (2005) 132.

[18] H. Touir, J. Dixmier, K. Zellama, J.F. Morhange, P. Alkaim, J. Non-Cryst. Solids 227 (1998) 906.

[19] R.J. Martín-Palma, L. Pascual, P. Herrero, J.M. Martínez-Duart, Appl. Phys. Lett. 87 (2005)

(211906-1-3).

[20] E. Edelberg, S. Bergh, R. Naone, M. Hall, E.S. Aydil, J. Appl. Phys. 81 (1997) 2410.

[21] R. Tsu, J. Gonzale Z-Hernandez, S.S. Chao, S.C. Lee, K. Tanaka, Appl. Phys. Lett. 40 (1982) 534.

[22] D. Gracin, S. Bernstorff, P. Dubček, A. Gajović, K. Juraić, Thin Solid Films 515 (2007) 5615.

[23] D. Gracin, K. Juraic, P. Dubček, A. Gajović, S. Bernstorff, Appl. Surf. Sci. 252 (2006) 5598.

[24] D. Gracin, S. Bernstorff, P. Dubček, A. Gajović, K. Juraić, J. Appl. Crystallogr. 40 (2007) s373s376.

[25] S. Manotas, F. Agulló-Rueda, J.D. Moreno, R.J. Martín-Palma, R. Guerrero-Lemus, J.M. MartínezDuart, Appl. Phys. Lett. 75 (1999)

977-979.

[26] R.J. Hill, C.J. Howard, J. Appl. Crystallogr. 467 (1987) 20.

[27] Statistica 7.1, StaSoft Inc. 1984-2005.

[28] I. Chambouleyron, S.D. Ventura, E.G. Birgin, J.M. Martínez-Duart, J. Appl. Phys. 92 (2002) 3093.

[29] T.D. Kang, H. Lee, S.J. Park, J. Jang, S. Lee, J. Appl. Phys. 92 (2002) 2467. 